\title{
PERFORMANCE OF HYBRID LEARNING CONTROL WITH INPUT SHAPING FOR INPUT TRACKING AND VIBRATION SUPPRESSION OF A FLEXIBLE MANIPULATOR
}

\author{
M. Z. Md Zain ${ }^{1}$, M. O. Tokhi ${ }^{1}$ and Z. Mohamed ${ }^{2}$ \\ ${ }^{1}$ Department of Automatic Control and Systems Engineering, The University of Sheffield, \\ UK. \\ ${ }^{2}$ Faculty of Electrical Engineering, University Technology Malaysia, Malaysia
}

\begin{abstract}
The objective of the work reported in this paper is to investigate the development of an intelligent hybrid iterative learning control scheme with input shaping for input tracking and end-point vibration suppression of a flexible manipulator. The dynamic model of the system is derived using the finite element method. Initially, a collocated proportional-derivative (PD) controller utilizing hub-angle and hub-velocity feedback is developed for control of rigidbody motion of the system. This is then extended to incorporate iterative learning control with genetic algorithm (GA) to optimize the learning parameters and a feedforward controller based on input shaping techniques for control of vibration (flexible motion) of the system. Simulation results of the response of the manipulator with the controllers are presented in time and frequency domains. The performance of the hybrid learning control with input shaping scheme is assessed in terms of input tracking and level of vibration reduction. The effectiveness of the control schemes in handling various payloads is also studied.
\end{abstract}

Keywords: Flexible manipulator, genetic algorithms, intelligent control, iterative learning control, input shaping.

\section{INTRODUCTION}

Most robot manipulators are designed and built in a manner to maximize stiffness, in an attempt to minimise system vibration and achieve good positional accuracy. High stiffness is achieved by using heavy material. As a consequence, such robots are usually heavy with 
respect to the operating payload. This, in turn, limits the speed of operation of the robot manipulation, increases the size of actuator, boosts energy consumption and increases the overall cost. Moreover, the payload to robot weight ratio, under such situations, is low. In order to solve these problems, robotic systems are designed to be lightweight and thus possess some level of flexibility.

Flexible manipulators exhibit many advantages over their rigid counterparts: they require less material, are lighter in weight, have higher manipulation speed, lower power consumption, require smaller actuators, are more maneuverable and transportable, are safer to operate due to reduced inertia, have enhanced back-drive ability due to elimination of gearing, have less overall cost and higher payload to robot weight ratio [1]. However, the control of flexible manipulators to maintain accurate positioning is an extremely challenging problem. Due to the flexible nature and distributed characteristics of the system, the dynamics are highly non-linear and complex. Problems arise due to precise positioning requirement, vibration due to system flexibility, difficulty in obtaining accurate model of the system and non-minimum phase characteristics [2,3]. In this respect, a control mechanism that accounts for both the rigid body and flexural motions of the system is required. If the advantages associated with lightness are not to be sacrificed, accurate models and efficient control strategies for flexible robot manipulators have to be developed.

The control strategies for flexible robot manipulator systems can be classified as feedforward (open-loop) and feedback (closed-loop) control schemes. Feed-forward techniques for vibration suppression involve developing the control input through consideration of the physical and vibrational properties of the system, so that system vibrations at response modes are reduced. This method does not require any additional sensors or actuators and does not account for changes in the system once the input is developed. On the other hand, feedbackcontrol techniques use measurement and estimations of the system states to reduce vibration. Feedback controllers can be designed to be robust to parameter uncertainty. For flexible 
manipulators, feedforward and feedback control techniques are used for vibration suppression and position control respectively. An acceptable system performance without vibration that accounts for system changes can be achieved by developing a hybrid controller consisting of both control techniques. Thus, a properly designed feedforward controller is required, with which the complexity of the required feedback controller can be reduced.

This paper presents investigations into the development of hybrid learning control with input shaping for input tracking and end-point vibration suppression of a flexible manipulator system. A constrained planar single-link flexible manipulator is considered and a simulation environment is developed within Simulink and Matlab for evaluation of performance of the control strategies. In this work, the dynamic model of the flexible manipulator is derived using the finite element (FE) method. Previous simulation and experimental studies have shown that the FE method gives an acceptable dynamic characterization of the actual system [4. Previously, a collocated PD control with a non-collocated PID control has been developed [5]. To demonstrate the effectiveness of the proposed control schemes, initially a joint-based collocated PD control utilising hub-angle and hub-velocity feedback is developed for control of rigid body motion of the manipulator. This is then extended to incorporate an iterative learning control (ILC) scheme, with genetic algorithms (GAs) for optimization of the learning parameters and input shaping for vibration suppression of the manipulator. For noncollocated control, end-point displacement feedback through a PID control configuration is developed whereas in the feedforward scheme, the input shaping technique is utilised as this has been shown to be effective in reducing system vibration [5]. Simulation results of the response of the manipulator with the controllers are presented in time and frequency domains. The performances of the hybrid learning control with input shaping are assessed in terms of input tracking and level of vibration reduction in comparison to the response with collocated PD and non-collocated PID (PD-PID) control. As the dynamic behaviour of the system changes with different payloads, the effectiveness of the controllers is also studied with a 
different loading condition. Finally, a comparative assessment of the hybrid learning control scheme in input tracking and vibration suppression of the manipulator is presented.

\section{THE FLEXIBLE MANIPULATOR SYSTEM}

A schematic representation of the single-link flexible manipulator system considered in this work is shown in Figure 1, where a control torque $\tau(t)$ is applied at the hub by an actuator motor, $E, I, \rho, L, I_{H}$ and $M_{p}$ represent Young's modulus, moment of inertia, mass density per unit volume, length, hub inertia and payload of the manipulator respectively. The angular displacement of the link in the POQ co-ordinates is denoted as $\theta(t) . w$ represents the elastic deflection of the manipulator at a distance $x$ from the hub, measured along the OP' axis. POQ and P'OQ' represent the stationary and moving frames respectively.

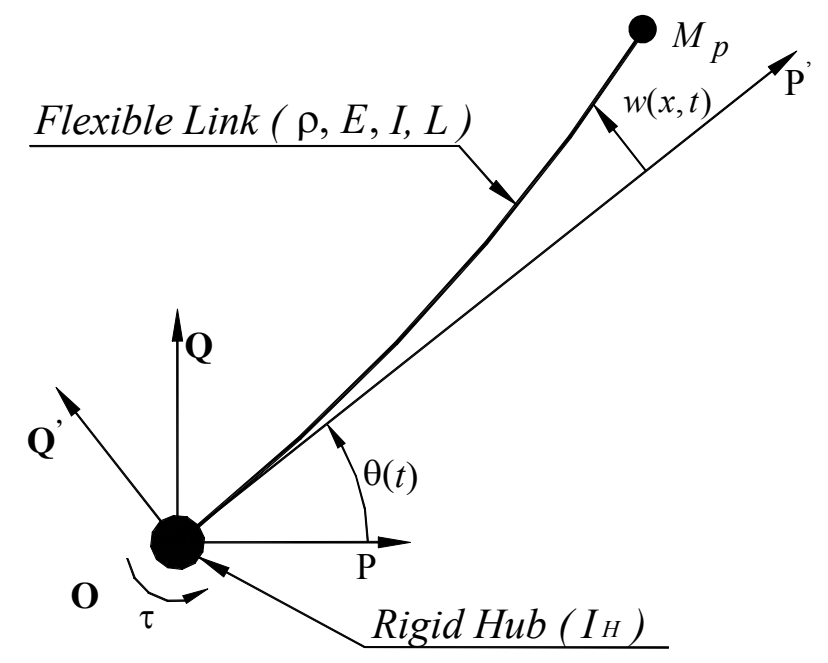

Figure 1: Schematic representation of the single-link flexible manipulator.

The height (width) of the link is assumed to be much greater than its depth, thus allowing the manipulator to vibrate dominantly in the horizontal direction (POQ plane). To avoid difficulties arising from time varying lengths, the length of the manipulator is assumed to be constant. Moreover, shear deformation, rotary inertia and the effect of axial force are ignored. 
For an angular displacement $\theta$ and an elastic deflection $w$, the total displacement $y(x, t)$ of a point along the manipulator at a distance $x$ from the hub can be described as a function of both the rigid body motion $\theta(t)$ and elastic deflection $w(x, t)$,

$$
y(x, t)=x \theta(t)+w(x, t)
$$

Thus, the net deflection at $x$ is the sum of a rigid body deflection and an elastic deflection. Note that by allowing the manipulator to be dominantly flexible in the horizontal direction the elastic deflection of the manipulator can be assumed to be confined to the horizontal plane only. In general, the motion of a manipulator will include elastic deflection in both, the vertical and horizontal planes. Motion in the vertical plane as a result of gravity forces for example, can cause permanent elastic deflections. This effect is neglected here as the manipulator is assumed to be dominantly flexible in the horizontal plane. In this study, an aluminium-type flexible manipulator of dimensions $900 \mathrm{~mm} \times 19.008 \mathrm{~mm} \times 3.2004 \mathrm{~mm}$, $E=71 \times 10^{9} \mathrm{~N} / \mathrm{m}^{2}, \quad I=5.253 \times 10^{-11} \mathrm{~m}^{4}$ and $I_{H}=5.8598 \times 10^{-4} \mathrm{kgm}^{2}$ is considered. A simulation algorithm characteristising the dynamic behaviour of the manipulator has previously been developed using the finite element (FE) method [4]. This is used in this work as a platform for test and evaluation of the proposed control approaches.

\section{CONTROL SCHEMES}

In this section, control schemes for rigid-body motion control and vibration suppression of the flexible manipulator are introduced. Initially, a collocated PD control is designed. This is then extended to incorporate an ILC scheme for control of vibration of the system.

\subsection{Collocated PD control}

A common strategy in the control of manipulator systems involves the utilization of PD feedback of collocated sensor signals. Such a strategy is adopted at this stage of the 
investigation here. A block diagram of the PD controller is shown in Figure 2, where $K_{p}$ and $K_{v}$ are the proportional and derivative gains respectively $\theta$ and $\dot{\theta}$ represent hub angle and hub velocity respectively, $R_{f}$ is the reference hub angle and $A_{c}$ is the gain of the motor amplifier. Here the motor/amplifier set is considered as a linear gain $A_{c}$. To design the PD controller a linear state-space model of the flexible manipulator was obtained by linearizing the equations of motion of the system. The first two flexible modes of the manipulator were assumed to be dominantly significant. The control signal $u(s)$ in Figure 2 can thus be written as

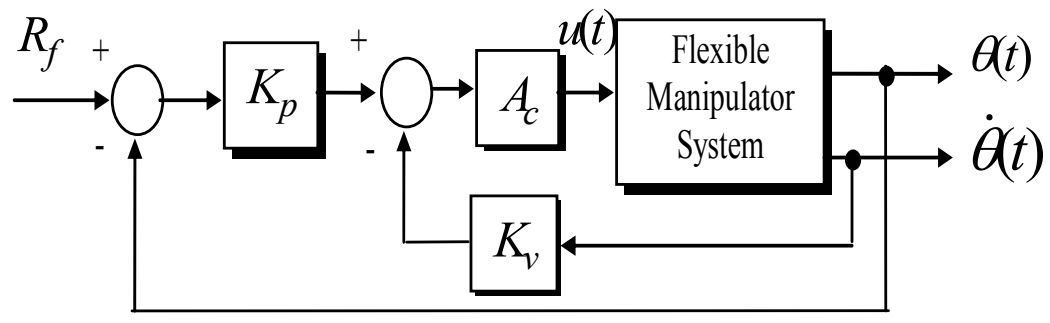

Figure 2: The collocated PD control structure

$$
u(s)=A_{c}\left[K_{p}\left\{R_{f}(s)-\theta(s)\right\}-K_{v} s \theta(s)\right]
$$

where $s$ is the Laplace variable. The closed-loop transfer function is, therefore, obtained as:

$$
\frac{\theta(s)}{R_{f}(s)}=\frac{K_{p} H(s) A_{c}}{1+A_{c} K_{v}\left(s+K_{p} / K_{v}\right) H(s)}
$$

where $H(s)$ is the open-loop transfer function from the input torque to hub angle, given by

$$
H(s)=\mathbf{C}(s \mathbf{I}-\mathbf{A})^{-1} \mathbf{B}
$$


where $\mathbf{A}, \mathbf{B}$ and $\mathbf{C}$ are the characteristic matrix, input matrix and output matrix of the system respectively and $\mathbf{I}$ is the identity matrix. The closed-loop poles of the system are, thus, given by the closed-loop characteristic equation as:

$$
1+K_{v}(s+Z) H(s) A_{c}=0
$$

where $Z=K_{p} / K_{v}$ represents the compensator zero which determines the control performance and characterises the shape of root locus of the closed-loop system. Theoretically any choice of the gains $K_{p}$ and $K_{v}$ assures the stability of the system [6]. In this study, the root locus approach is utilized to design the PD controller. Analyses of the root locus plot of the system show that dominant poles with maximum negative real parts could be achieved with $Z \approx 2$ and by setting $K_{p}$ between 0 and 1.2 [7].

\subsection{Hybrid Collocated PD with Iterative Learning Control}

A hybrid collocated PD control structure for control of rigid-body motion of the flexible manipulator with ILC is proposed in this section. In this study, an ILC scheme is developed using PD-type learning algorithm.

Iterative learning control has been an active research area for more than a decade, mainly inspired by the pioneering work of Arimoto et al. [8-10]. Learning control begun with the fundamental principle that repeated practice is a common mode of human learning. Given a goal (regulation, tracking, or optimization), learning control, or more specifically, iterative learning control refers to the mechanism by which necessary control can be synthesized by repeated trials. Moore [11] describes ILC as an approach to improving the transient response performance of a system that operates repetitively over a fixed interval. This is especially applicable to a system such as industrial robot which accomplishes most of its tasks repetitively over a period of time. Consider a robot arm in which a number of conditions such 
as varying the input parameters and disturbances, are imposed. Performance of the arm, e.g. trajectory control can be evaluated, changed or improved iteratively by means of using the previous response. This is in turn incorporated in the control strategy during the next cycle to improve its performance. In this way, an ILC scheme is established in which unlike conventional adaptive control approaches, the control strategy is changed by changing the command reference signal and not the controller itself. Uchiyama first introduced the concept of iterative learning for generating the optimal input to a system [12]. Arimoto and his coworkers later developed the idea [8-10].

Figure 3 illustrates the basic idea of ILC. The input signal $\Psi_{k}(t)$ and output signal $x_{k}(t)$, are stored in memory (some type of memory device is implicitly assumed in the block labeled "learning controller"). By using the desired output of the system $x_{d}(t)$ and the actual output $x_{k}(t)$, the performance error at $k$ th trial can be defined as:

$$
e_{k}(t)=x_{d}(t)-x_{k}(t)
$$

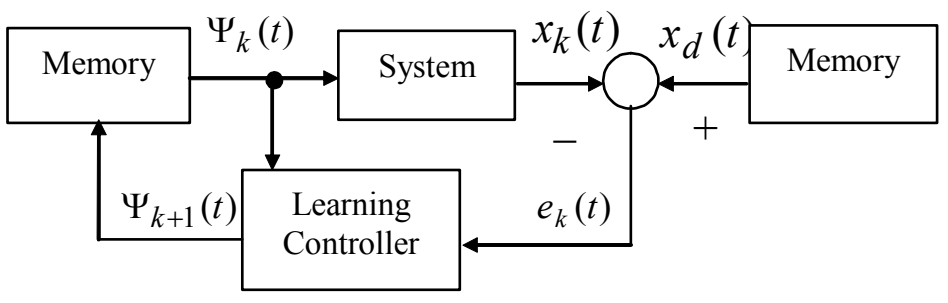

Figure 3: Iterative learning control configuration

The aim of ILC is to iteratively compute a new compensation input signal $\Psi_{k+1}(t)$, which is stored for use in the next trial. The next input command is chosen in such a way as to guarantee that the performance error will be reduced in the next trial. The important task in the design of a learning controller is to find an algorithm for generating the next input in such a way that the performance error is reduced on successive trials. In other words, the algorithm 
needs to lead to the convergence of the error to minimum. Another consideration is that it is desirable to have the convergence of the error without or at least with minimal knowledge of the model of the system. Further, the algorithm should be independent of the functional form of the desired response, $x_{d}(t)$. Thus, the learning controller would "learn" the best possible control signal for a particular desired output trajectory even if it is newly introduced without the need to reconfigure the algorithm.

In this work a learning algorithm of the following form is considered:

$$
\Psi_{k+1}=\Psi_{k}+\Phi e_{k}+\Gamma \dot{e}_{k}
$$

where

$\Psi_{k+1}$ is the next control signal

$\Psi_{k} \quad$ is the current control signal

$e_{k}$ is the current positional error input, $e_{k}=\left(x_{d}-x_{k}\right) . \Phi, \Gamma$ are suitable positive definite constants (or learning parameters)

A block diagram of the scheme is shown in Figure 4. It is obvious that the algorithm contains a constant and derivative coefficient of the error. In other words, the expression can be simply called proportional-derivative or PD type learning algorithm. A slightly modified learning algorithm to suit the application is employed here. Instead of using the absolute position tracking error $e_{k}$, a sum-squared tracking error $e_{k}$ is used. Figure 4 shows a block diagram describing the above expression. This is used with PD collocated control, to realise the hybrid collocated PD with ILC. This is shown in Figure 5. 


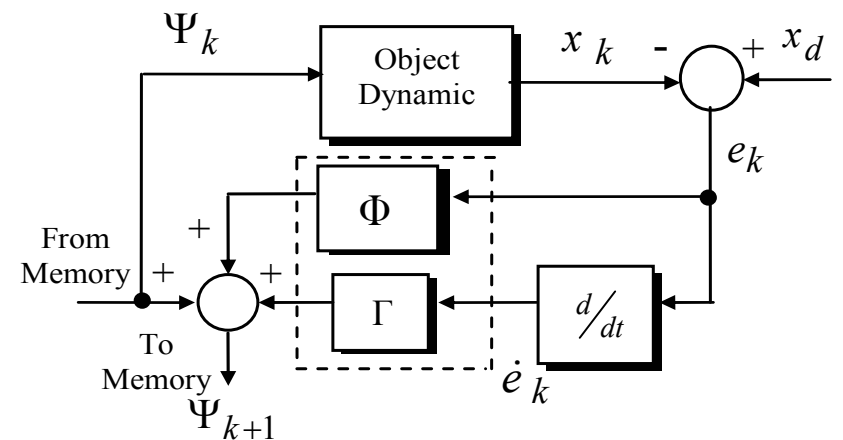

Figure 4: PD type learning algorithm

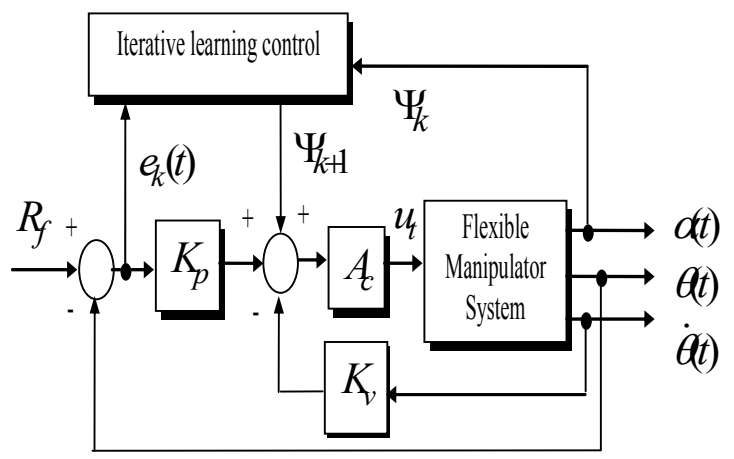

Figure 5: The collocated PD with iterative learning control structure

\subsection{GA based hybrid learning control}

Figure 4 shows the PD-type learning control scheme. The performance of the PD-type learning control depends upon the proportional gain $\Phi$ and derivative gain $\Gamma$. Stability, settling time, maximum overshoot and many other system performance indicators depend upon the values of $\Phi$ and $\Gamma$. The proposed strategy utilizes GA as an optimization and search tool to determine optimal values for the gains. The performance index or the cost function chosen is the error taken by the system to reach and stay within a range specified by absolute percentage of the final value. Hence, the role of GA is to find optimum vales of the gains $\Phi$ and $\Gamma$. In this case, integral of absolute error (IAE) is used for minimizing the error and generating the controller parameters:

$$
I A E=\int_{0}^{T}\left|\frac{\sum \text { Error }^{2}}{N}\right| d t
$$

where, Error $=r(t)-y(t), N=$ size of sample,$r(t)=$ reference input, $y(t)=$ measured variable. Thus, the function in equation (8) can be minimized by applying a suitable tuning algorithm as illustrated in the next section or through the application of a genetic algorithm. The GA used here initializes a random set of population of the two variables $\Phi$ and $\Gamma$. The 
algorithm evaluates all members of the population based on the specified performance index. The algorithm then applies the GA operators such as reproduction, crossover and mutation to generate a new set of population based on the performance of the members of the population [13]. The best member or gene of the population is chosen and saved for next generation. It again applies all operations and selects the best gene among the new population. The best gene of the new population is compared to best gene of previous population. If a predefined termination criterion is not met, a new population is obtained in the same way as above. The termination criterion may be formulated as the magnitude of difference between index value of previous generation and present generation becoming less than a prespecified value. The process continues till the termination criterion is fulfilled.

\subsection{Hybrid PD and non-collocated control}

The use of a non-collocated control, where the end-point of the manipulator is controlled by measuring its position, can be applied to improve the overall performance, as more reliable output measurement can be obtained. The control structure comprises two feedback loops: a) the hub-angle and hub- velocity as inputs to a collocated control law for rigid-body motion control; b) the end-point residual (elastic deformation) as input to a separate non-collocated control law for vibration control. These two loops are then summed together to give a torque input to the system. A block diagram of the control scheme is shown in Figure 6 , where $r_{\alpha}$ represents the end-point residual reference input, which is set to zero as the control objective is to have zero vibration during movement of the manipulator. For rigid-body motion control, the PD control strategy developed in the previous section is adopted whereas for the vibration control loop, the end-point residual feedback through a PID control scheme is utilized. The values of proportional (P), derivative (D) and integral (I) gains are adjusted using the ZiegelNichols procedure [14]. For the two control loops to work well they have to be decoupled 
from one another. This can be achieved by using a high-pass filter in the non-collocated control loop.

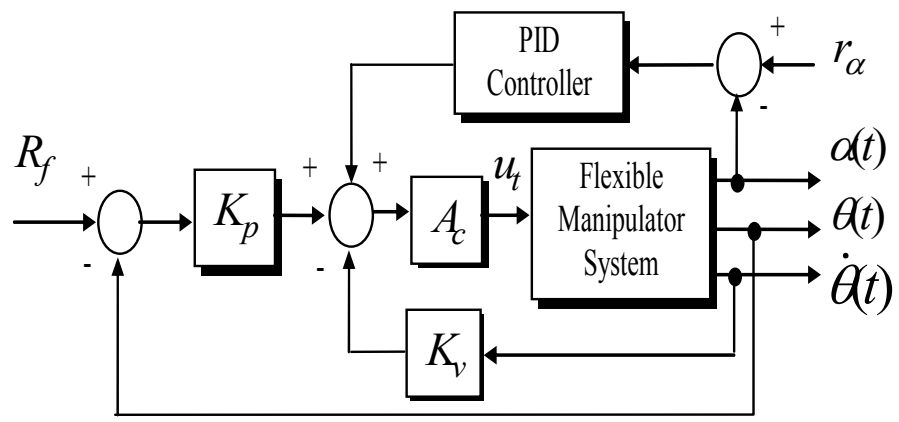

Figure 6: The collocated PD and non-collocated PID control

\subsection{Hybrid control with input shaping}

The method of input shaping involves convolving a desired command with a sequence of impulses. The design objectives are to determine the amplitude and time location of the impulses. A brief derivation is given below. Further details can be found in [15]. A vibratory system of any order can be modelled as a superposition of second order systems with transfer function

$$
G(s)=\frac{\omega^{2}}{s^{2}+2 \xi \omega s+\omega^{2}}
$$

where $\omega$ is the natural frequency and $\xi$ is the damping ratio of the system. Thus, the impulse response of the system can be obtained as:

$$
y(t)=\frac{A \omega}{\sqrt{1-\xi^{2}}} e^{-\xi \omega\left(t-t_{o}\right)} \sin \left(\omega \sqrt{1-\xi^{2}}\left(t-t_{o}\right)\right)
$$

where $A$ and $t_{0}$ are the amplitude and time location of the impulse respectively. Further, the response to a sequence of impulses can be obtained by superposition of the impulse 
responses. Thus, for $N$ impulses, with $\omega_{d}=\omega\left(\sqrt{1-\xi^{2}}\right)$, the impulse response can be expressed as:

$$
y(t)=M \sin \left(\omega_{d} t+\beta\right)
$$

where

$$
M=\sqrt{\left(\sum_{i=1}^{N} B_{i} \cos \phi_{i}\right)^{2}+\left(\sum_{i=1}^{N} B_{i} \sin \phi_{i}\right)^{2}}, B_{i}=\frac{A_{i} \omega}{\sqrt{1-\xi^{2}}} e^{-\xi \omega\left(t-t_{o}\right)}, \phi_{i}=\omega_{d} t_{i}
$$

$A_{i}$ and $t_{i}$ are the magnitudes and time locations of the impulses.

The residual vibration amplitude of the impulse response can be obtained by evaluating the response at the time of the last impulse, $t_{N}$ as:

$$
V=\frac{\omega}{\sqrt{1-\xi^{2}}} e^{-\xi \omega\left(t_{N}\right)} \sqrt{(C(\omega, \xi))^{2}+(S(\omega, \xi))^{2}}
$$

where

$$
C(\omega, \xi)=\sum_{i=1}^{N} A_{i} e^{-\xi \omega t_{i}} \cos \left(\omega_{d} t_{i}\right)
$$

and

$$
S(\omega, \xi)=\sum_{i=1}^{N} A_{i} e^{-\xi \omega t_{i}} \sin \left(\omega_{d} t_{i}\right)
$$

In order to achieve zero vibration after the input has ended, it is required that $C(\omega, \xi)$ and $S(\omega, \xi)$ in equation (9) are independently zero. Furthermore, to ensure that the shaped command input produces the same rigid body motion as the unshaped command, it is 
required that the sum of impulse amplitudes, 3 unity, i.e. $\sum_{i=1}^{N} A_{i}=1$. To avoid delay, the first impulse is selected at time 0 . The simplest constraint is zero vibration at expected frequency and damping of vibration using a two-impulse sequence. Hence by setting equation (9) to zero, and solving yields a two-impulse sequence with parameters as:

$$
\begin{aligned}
& t_{1}=0, \quad t_{2}=\frac{\pi}{\omega_{d}} \\
& A_{1}=\frac{1}{1+K}, \quad A_{2}=\frac{K}{1+K}
\end{aligned}
$$

where

$$
K=e^{-\frac{\xi \pi}{\sqrt{1-\xi^{2}}}} .
$$

The robustness of the input shaper to error in natural frequencies of the system can be increased by setting $\frac{d V}{d \omega}=0$, where $\frac{d V}{d \omega}$ is the rate of change of $V$ with respect to $\omega$. Setting the derivative to zero is equivalent to setting small changes in vibration for changes in the natural frequency. Thus, additional constraints are added into the equation, which after solving yields a three-impulse sequence with parameters as:

$$
\begin{gathered}
t_{1}=0, \quad t_{2}=\frac{\pi}{\omega_{d}}, \quad t_{3}=2 t_{2}, \\
A_{1}=\frac{1}{1+2 K+K^{2}}, \quad A_{2}=\frac{2 K}{1+2 K+K^{2}}, \quad A_{3}=\frac{K^{2}}{1+2 K+K^{2}}
\end{gathered}
$$

where $K$ is as in equation (10). The robustness of the input shaper can further be increased by taking and solving the second derivative of the vibration in equation (9). Similarly, this yields a four-impulse sequence with parameters as: 


$$
\begin{gathered}
t_{1}=0, \quad t_{2}=\frac{\pi}{\omega_{d}}, \quad t_{3}=2 t_{2}, \quad t_{4}=3 t_{2}, \\
A_{1}=\frac{1}{1+3 K+3 K^{2}+K^{3}}, \quad A_{2}=\frac{3 K}{1+3 K+3 K^{2}+K^{3}}, \\
A_{3}=\frac{3 K^{2}}{1+3 K+3 K^{2}+K^{3}}, \quad A_{4}=\frac{K^{3}}{1+3 K+3 K^{2}+K^{3}}
\end{gathered}
$$

where $K$ is as in equation (10).

To handle higher vibration modes, an impulse sequence for each vibration mode can be designed independently. Then the impulse sequences can be convoluted together to form a sequence of impulses that attenuates vibration at higher modes. For any vibratory system, the vibration reduction can be accomplished by convolving any desired system input with the impulse sequence. This yields a shaped input that drives the system to a desired location without vibration. Incorporating the input shaping into PD-ILC structure results in the combined PD-ILC and input shaping control structure shown in Figure 7.

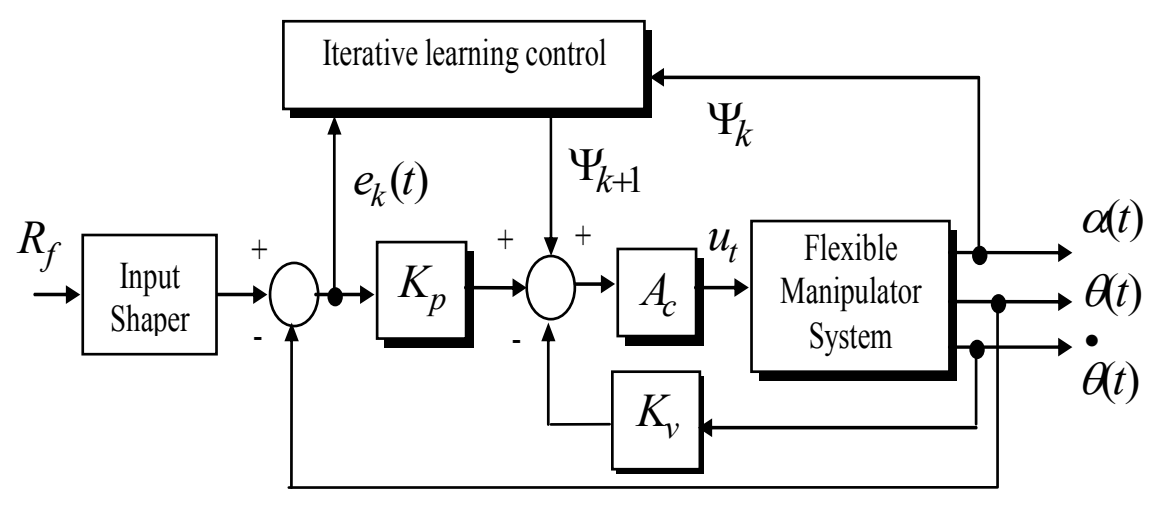

Figure 7: The PDILC control with input shaping structure

\section{SIMULATION RESULTS AND DISCUSSION}

In this section, the proposed control schemes are implemented and tested within the simulation environment of the flexible manipulator and the corresponding results are presented. The manipulator is required to follow a trajectory at $\pm 80^{\circ}$ as shown in Figure 8 . System responses, namely torque input, hub-angle and end-point residual are observed. To 
assess the vibration reduction in the system in the frequency domain, power spectral density (SD) of response at the end-point is obtained. Thus, the first three modes of vibration of the systems are considered as these dominantly characterise the behaviour of the manipulator. Figures 9 and 10 show simulated response of the manipulator at the end-point. Note that vibration occurs during movement of the manipulator and the end-point residual response oscillators between $\pm 3.53 \mathrm{~m}$ without payload and $\pm 3.16 \mathrm{~m}$ with a $15 \mathrm{~g}$ payload. The vibration frequencies of the system were obtained as 13, 35 and $65 \mathrm{~Hz}$ without payload and 12,33 and $60 \mathrm{~Hz}$ with a $15 \mathrm{~g}$ payload. These results were considered as the system response in open loop and subsequently used to design and evaluate the closed loop techniques.

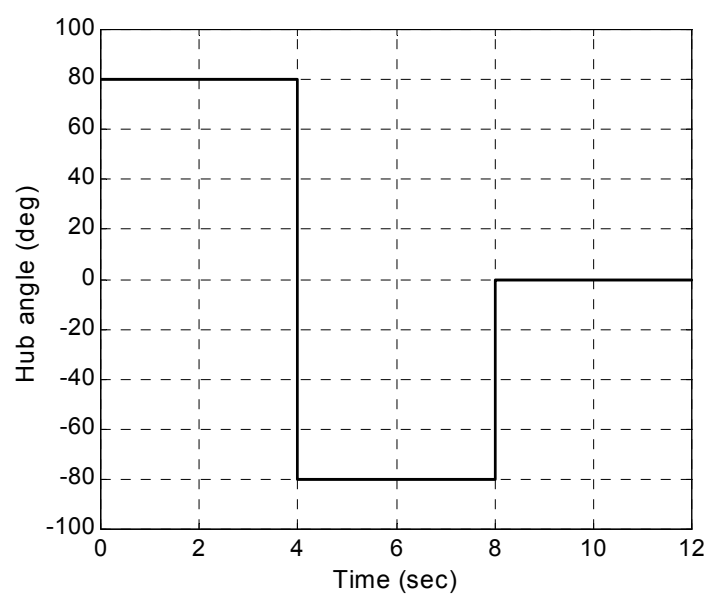

Figure 8: The reference hub angle

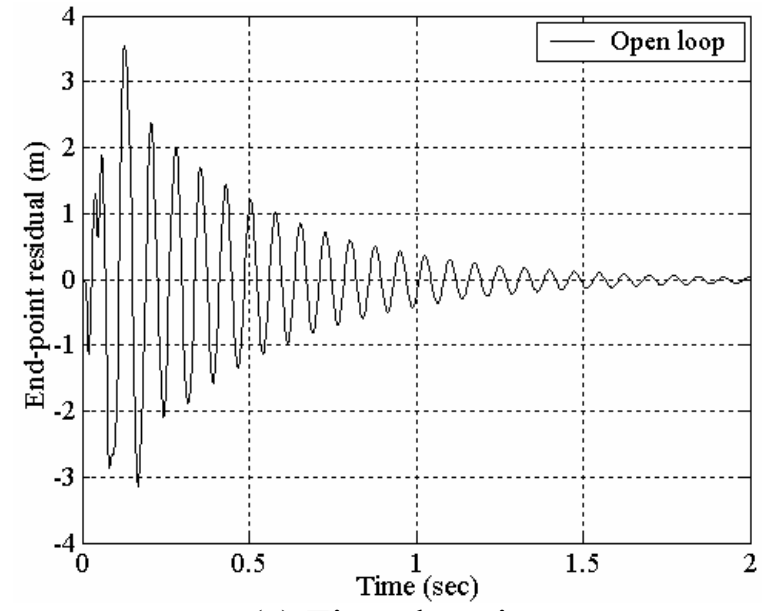

(a) Time domain

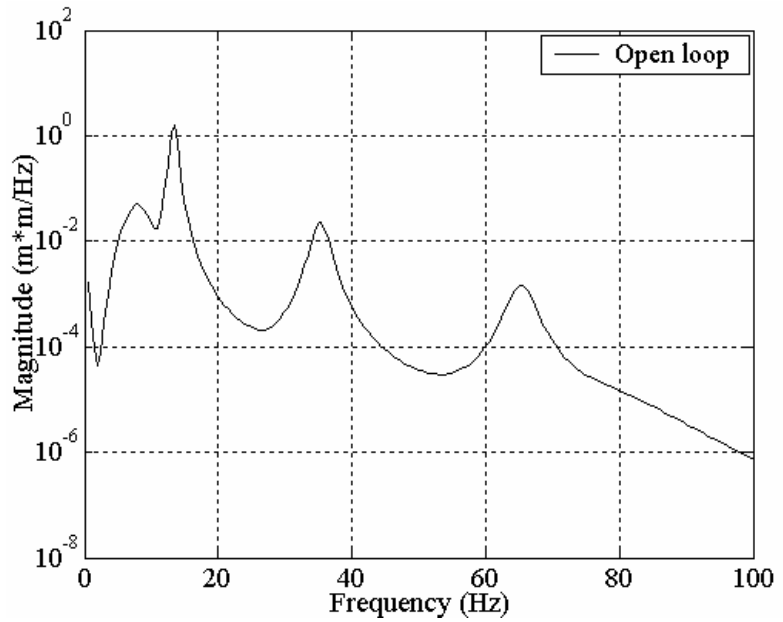

(b) Spectral density 
Figure 9: Response of the open loop end-point residual without payload

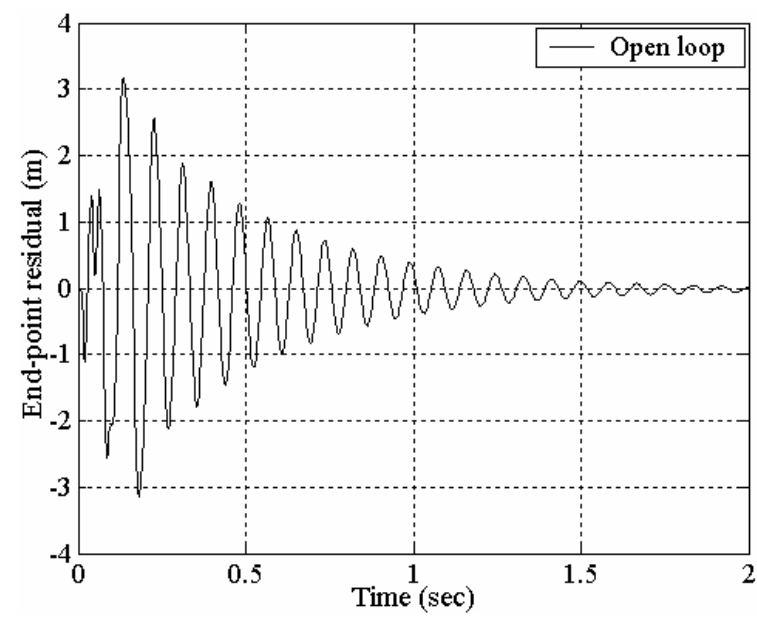

(a) Time domain

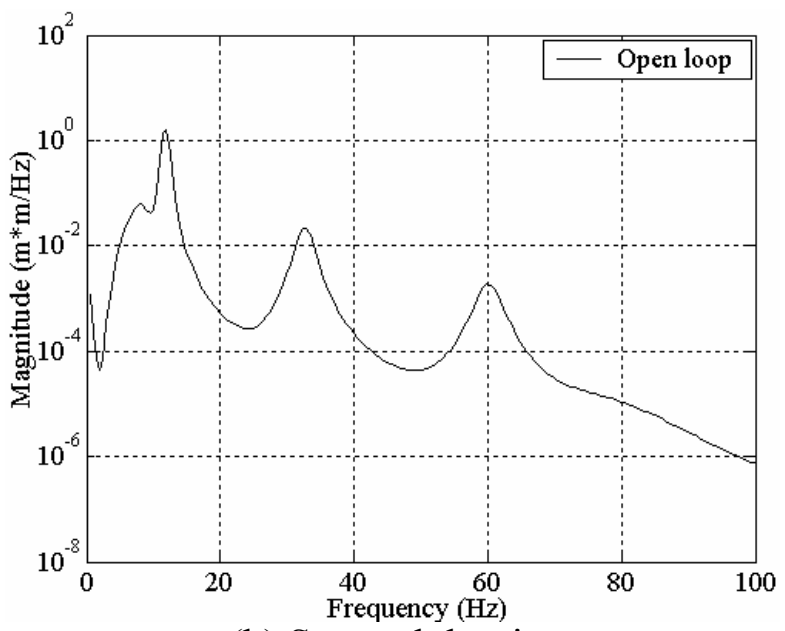

(b) Spectral density

Figure 10: Response of the open loop end-point residual with a $15 \mathrm{~g}$ payload

In the collocated and non-collocated control scheme of PD-PID (PDPID), the design of PD controller was based on root locus analysis, from which $K_{p}, K_{v}$ and $A_{c}$ were deduced as $0.64,0.32$ and 0.01 respectively. The required torque input driving the manipulator without payload with the collocated PD control is shown in Figure 11 (a). The corresponding system response is shown in Figure 11 (b),(c) and (d). The closed-loop parameters with the PD control will subsequently be used to design and evaluate the performance of non-collocated and feedforward control schemes in terms of input tracking capability and level of vibration reduction. The results in Figure 11 for the collocated PD control will be used for comparative assessment of the hybrid control schemes proposed in section 3 . 


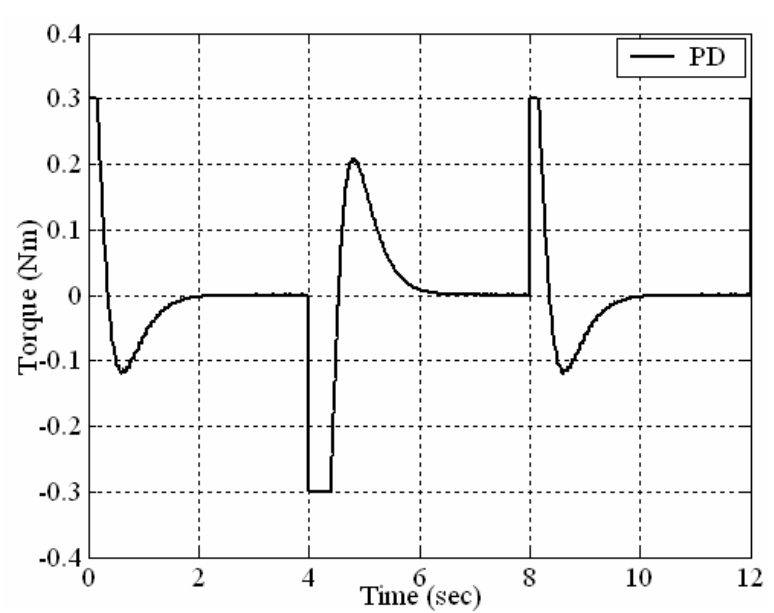

(a) Torque input (Time domain)

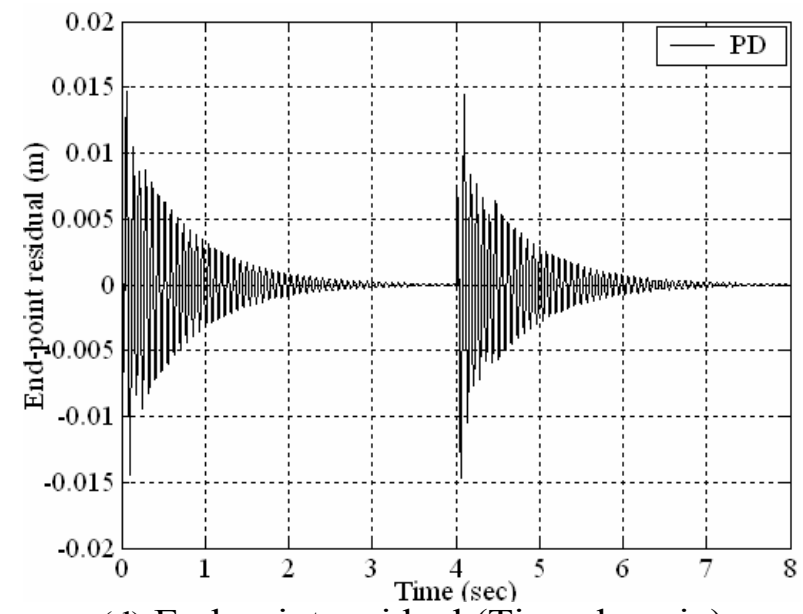

(d) End-point residual (Time domain)

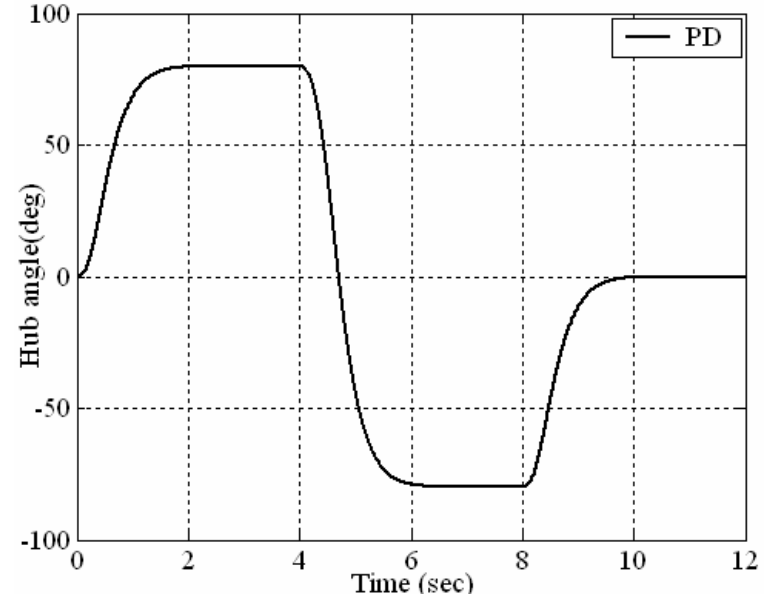

(b) Hub angle (Time domain)

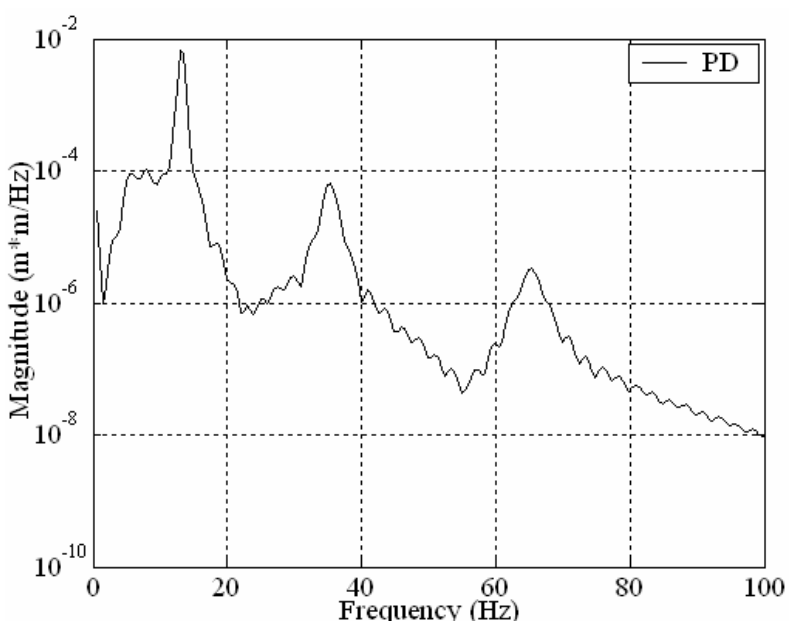

(d) Spectral density of end-point residual

Figure 11: Response of the manipulator with PD control

The PID controller parameters were tuned using the Ziegel-Nichols method using a closed-loop technique where the proportional gain $k_{p}$ was initially tuned and the integral gain $k_{i}$ and derivative gain $k_{d}$ were then calculated [14]. Accordingly, the PID parameters $k_{p}, k_{i}$ and $k_{d}$ were deduced as $0.1,70$ and 0.01 respectively. The corresponding system response with the PD-PID control is shown in Figures 12 and 13. It is noted that the manipulator reached the required position of $\pm 80^{0}$ within $2 \mathrm{~s}$, with no significant overshoot. However, a noticeable amount of vibration occurs during movement of the manipulator. It is noted from the end-point residual that the vibration of the system settles within $4 \mathrm{~s}$ with a maximum residual of $\pm 0.015 \mathrm{~m}$. Moreover, the vibration at the end-point was dominated by 
the first three vibration modes, which are obtained as $13 \mathrm{~Hz}, 35 \mathrm{~Hz}$ and $65 \mathrm{~Hz}$ without payload and $12 \mathrm{~Hz}, 33 \mathrm{~Hz}$ and $60 \mathrm{~Hz}$ with a 15 g payload. The flexible manipulator is set with a structural damping of $0.026,0.038$ and 0.05 for the first, second and third vibration modes respectively.

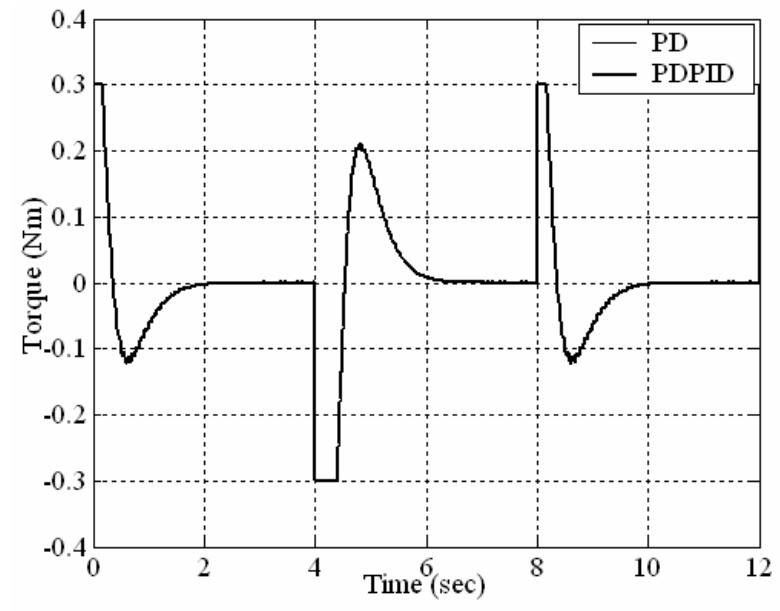

(a) Torque input (Time domain)

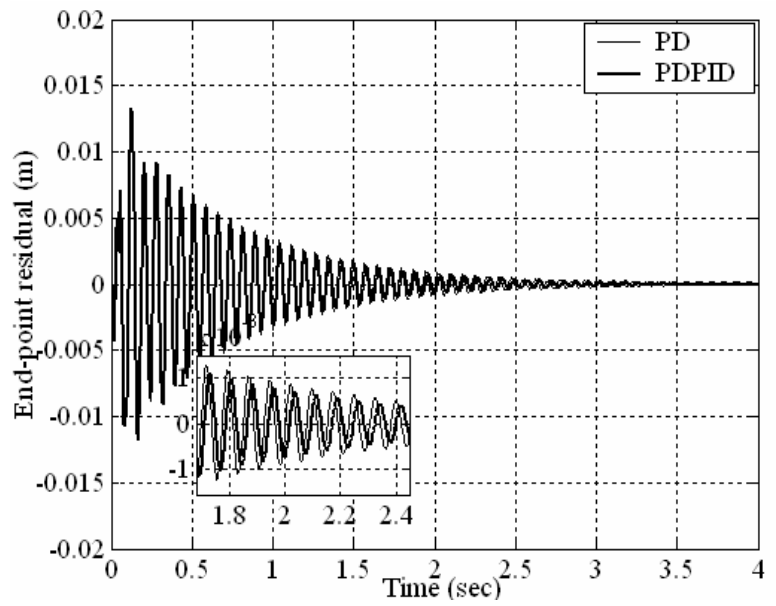

(c) End-point residual (Time domain)

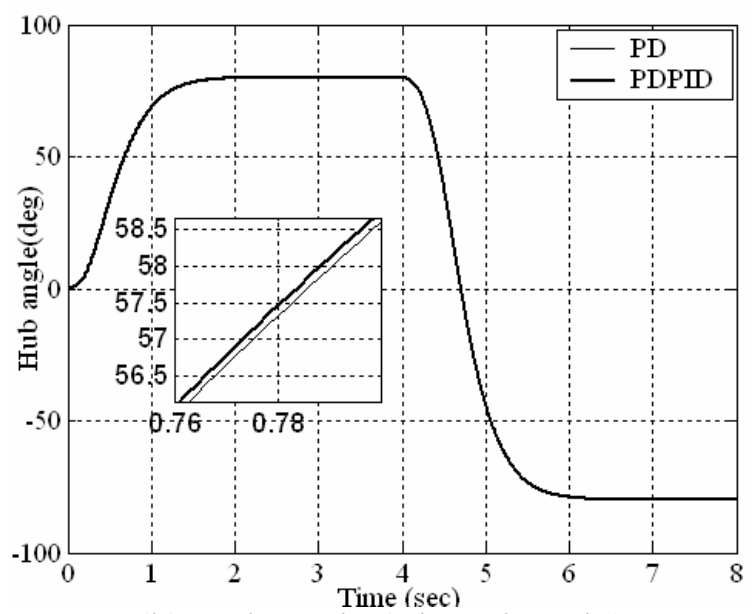

(b) Hub angle (Time domain)

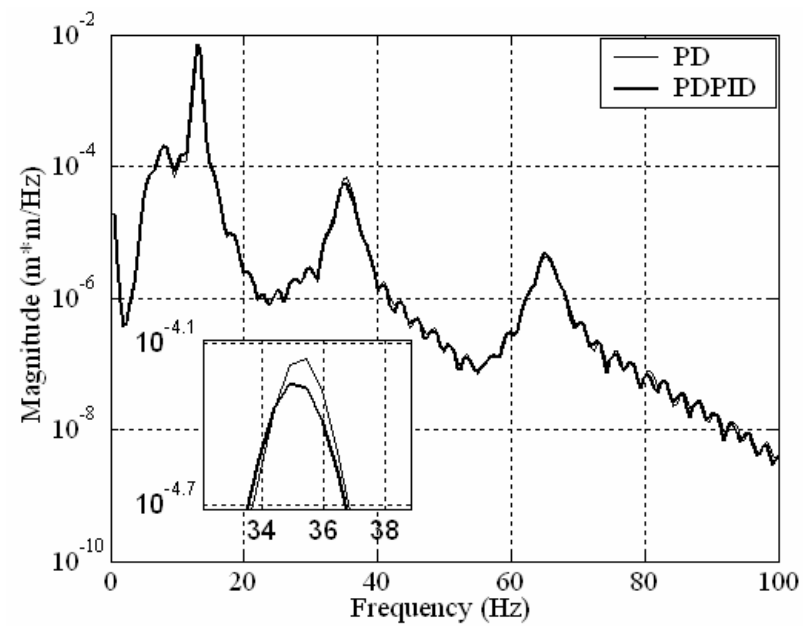

(d) Spectral density of end-point residual

Figure 12: Response of the manipulator with PD and PD-PID control without payload 


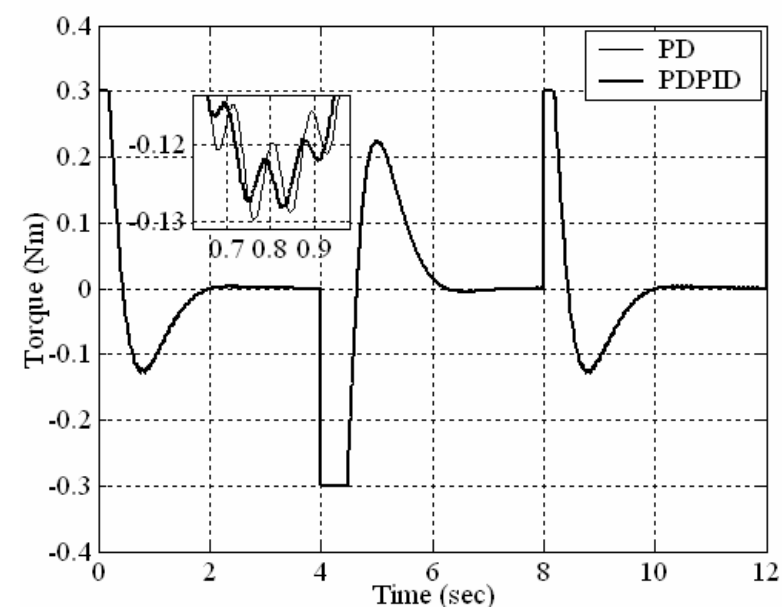

(a) Torque input (Time domain)

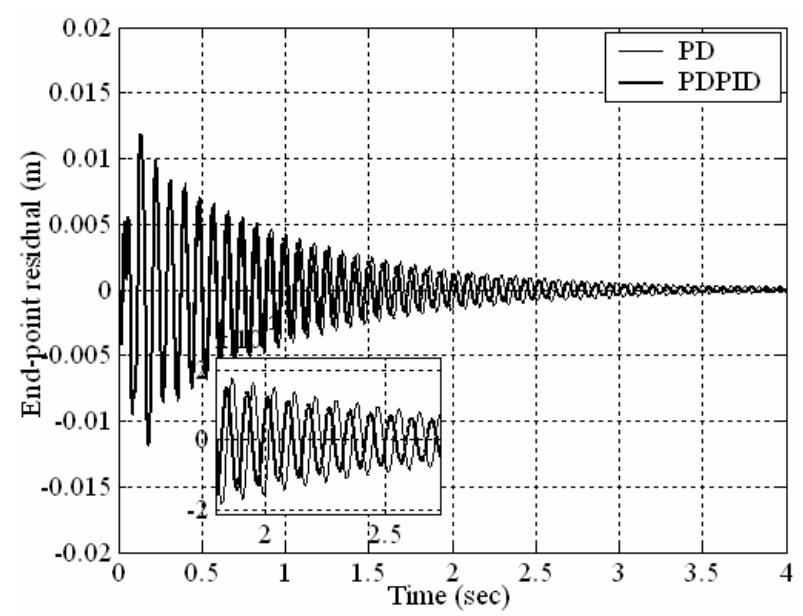

(c) End-point residual (Time domain)

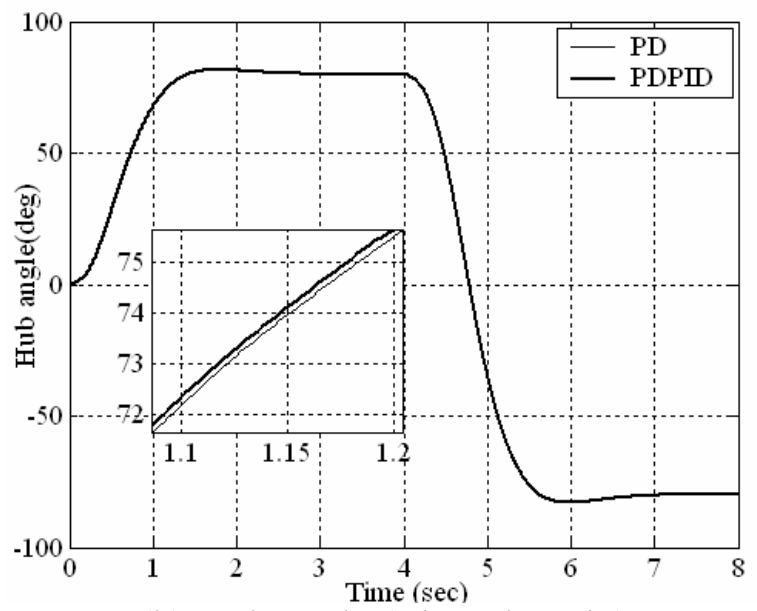

(b) Hub angle (Time domain)

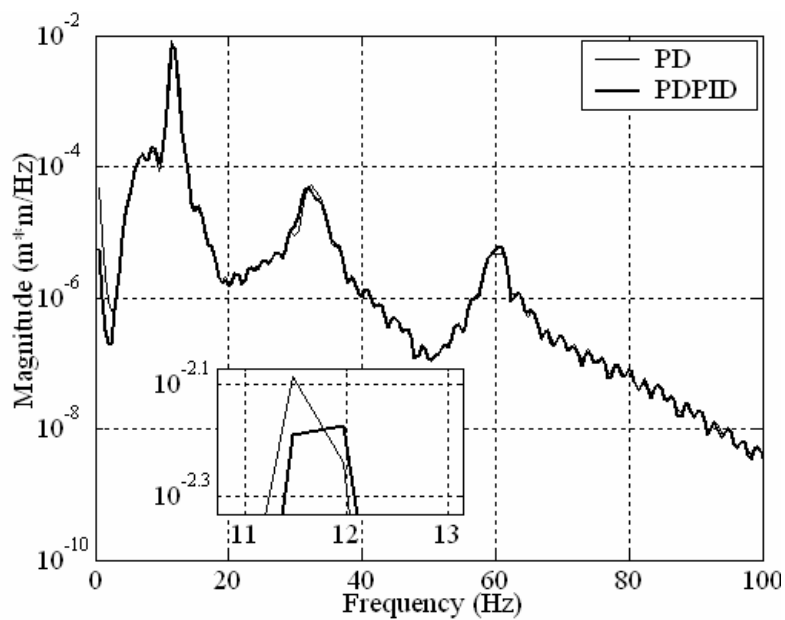

(d) Spectral density of end-point residual

Figure 13: Response of the manipulator with PD and PD-PID control with15 g payload

The (PD-ILC) scheme, was designed on the basis of the dynamic behaviour of the closed-loop system. The parameters of the learning algorithm, $\Phi$ and $\Gamma$ were tuned based on GA over the simulation period and were deduced as 0.0015 and 0.0011 respectively. The GA was designed with 80 individuals in each generation. The maximum number of generations was set to 100 . The algorithm achieved an IAE level of 0.0027783 in the $70^{\text {th }}$ generation. Figure 14 and Table 1 show the algorithm convergence as a function of generations and the parameter values used in the GA respectively. Figures 15 and 16 show the corresponding responses of the manipulator without payload and with a $15 \mathrm{~g}$ payload with PD-ILC. It is noted that the proposed hybrid controller with learning algorithm is capable of reducing the 
system vibration while resulting in better input tracking performance of the manipulator. The vibration of the system settled within less than $1.5 \mathrm{~s}$, which is much less than that achieved with PD-PID control. The closed-loop system parameters with the PD control will subsequently be used to design and evaluate the performance of ILC and feedforward control schemes in terms of input tracking capability and level of vibration reduction.

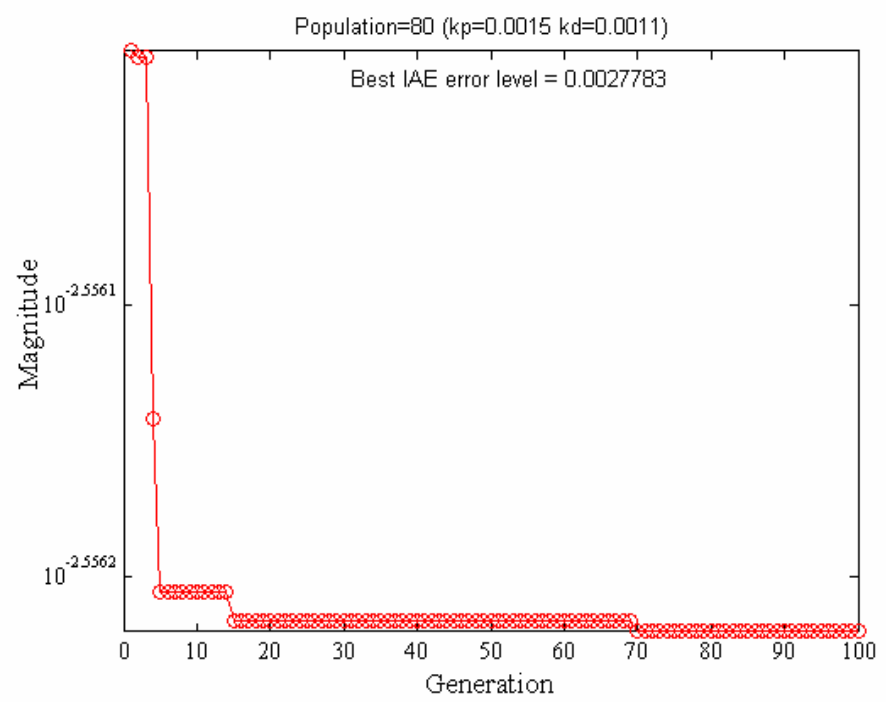

Figure 14: Objective value vs. number of generation 


\begin{tabular}{|c|c|}
\hline Parameter & Setting \\
\hline Generation gap & 0.9 \\
\hline Precision & 14 \\
\hline Crossover rate & 0.8 \\
\hline Mutation rate & 0.025 \\
\hline
\end{tabular}

Table 1: Algorithm parameter for PD-type learning

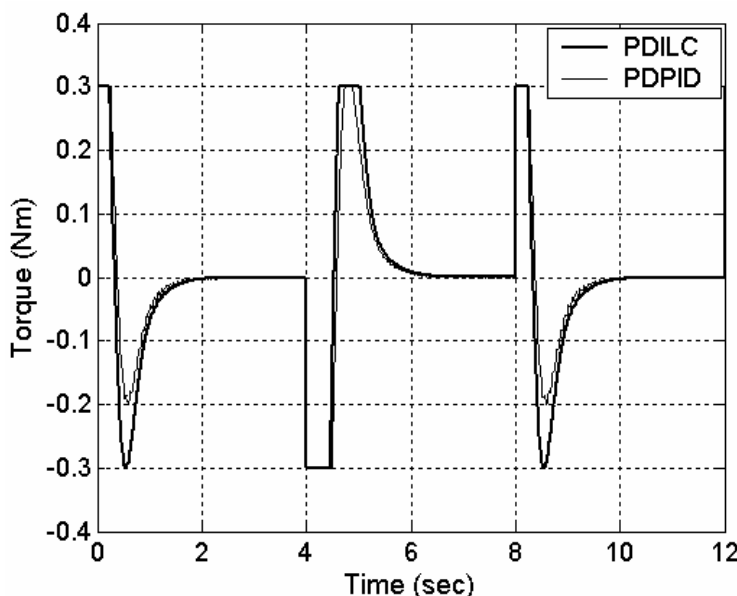

(a) Torque input (Time domain)

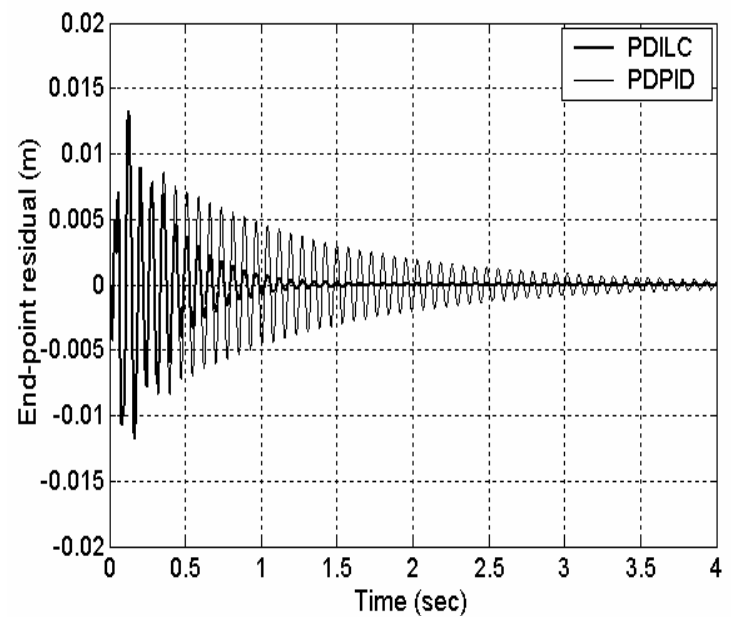

(c) End-point residual (Time domain)

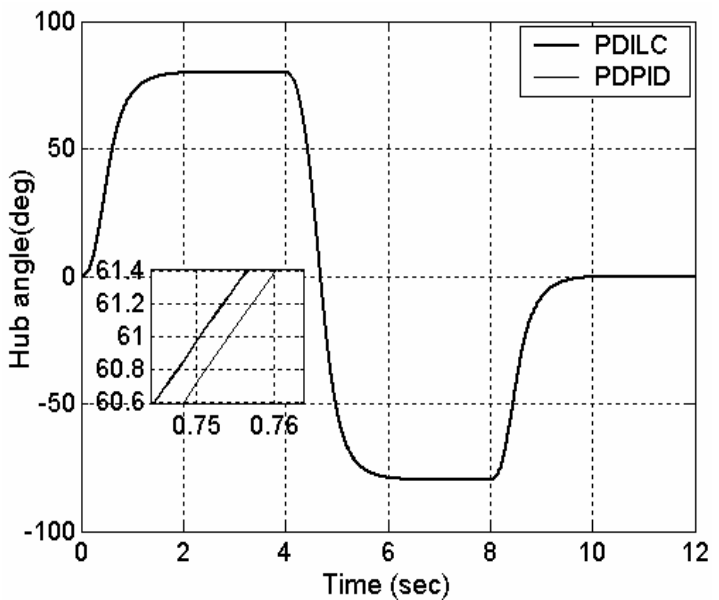

(b) Hub angle (Time domain)

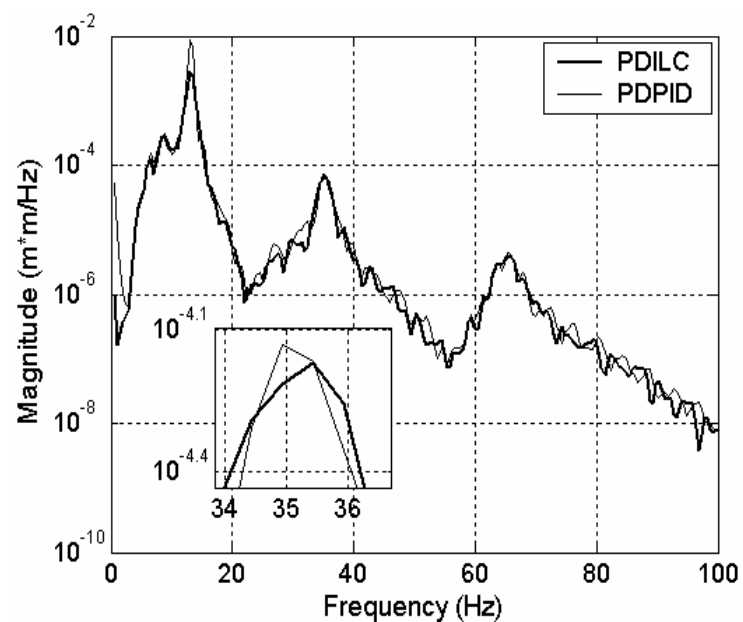

(d) Spectral density of end-point residual

Figure 15: Response of the manipulator with PD-ILC and PD-PID control without payload 


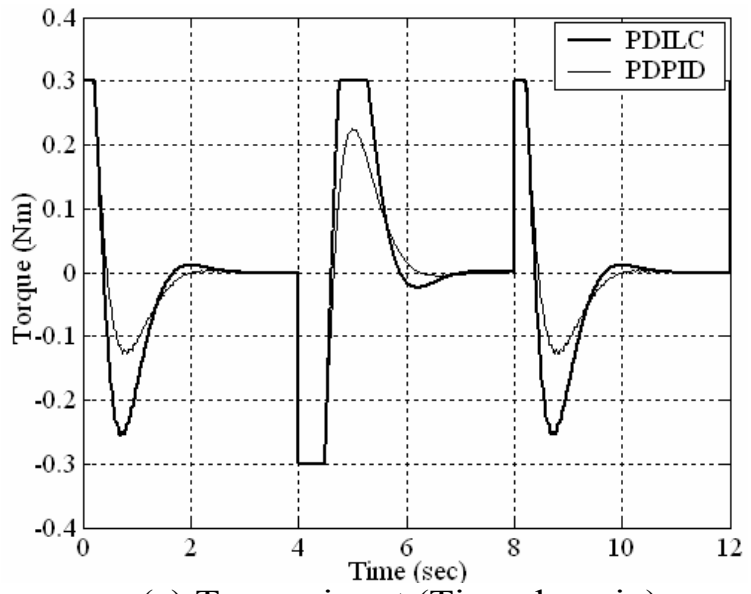

(a) Torque input (Time domain)

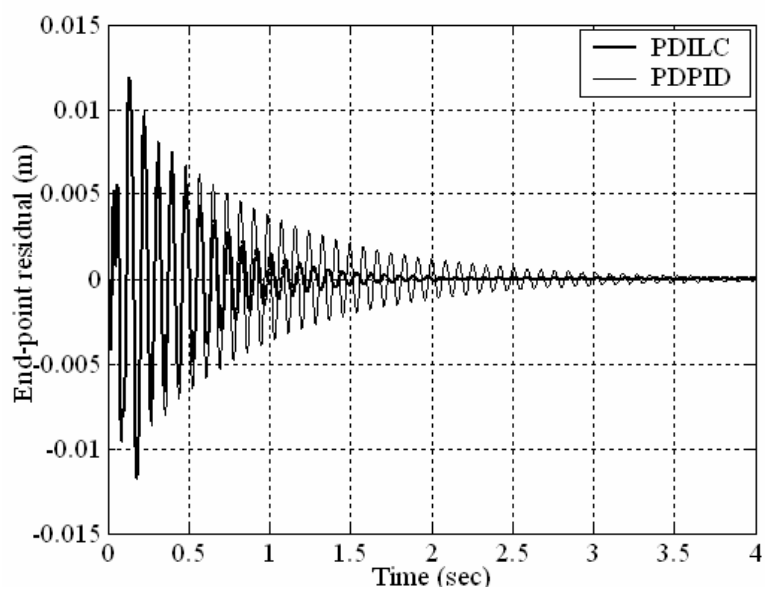

(c) End-point residual (Time domain)

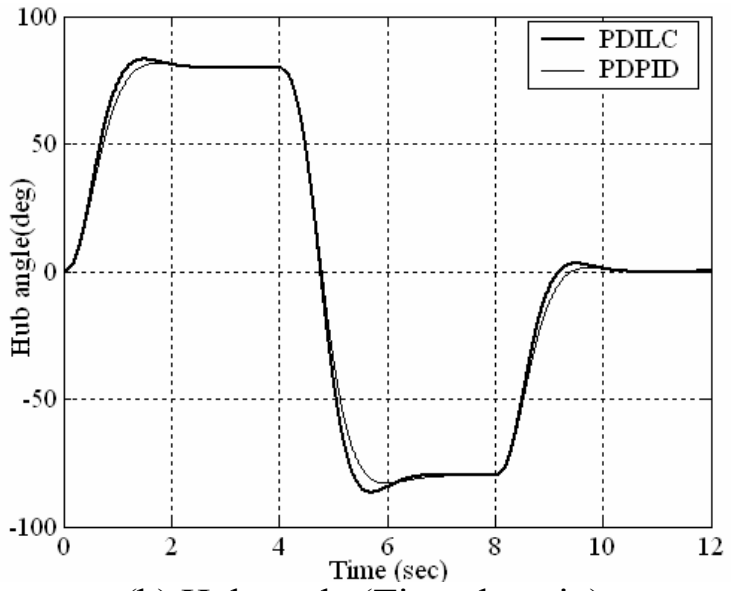

(b) Hub angle (Time domain)

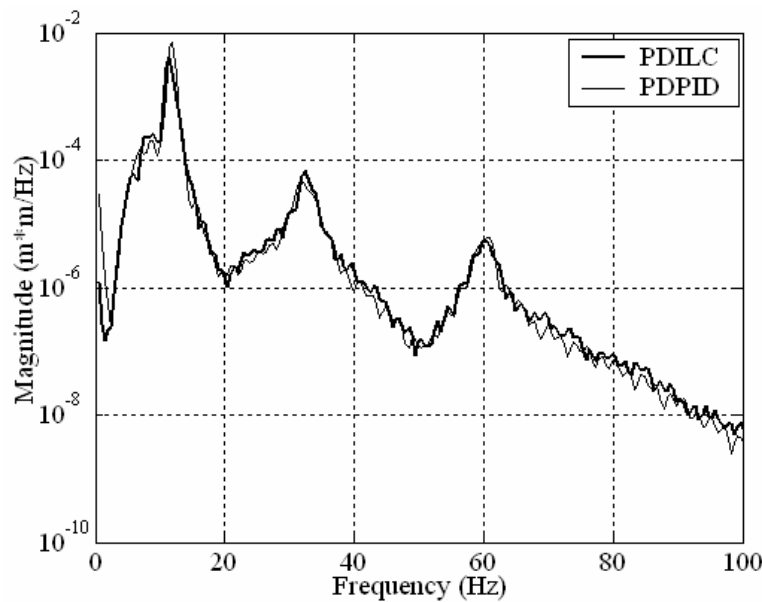

(d) Spectral density of end-point residual

Figure 16: Response of the manipulator with PD-ILC and PD-PID control with a $15 \mathrm{~g}$ payload

In the case of the hybrid learning and feedforward control scheme (PD-ILC-IS), an input shaper was designed based on the dynamic behaviour of the closed-loop system obtained using only the PD control. Figures 17 and 18 show the corresponding responses of the manipulator without payload and a 15 g payload with PD-PID and PD-ILC-IS. As shown in the previous section, the natural frequencies of the manipulator were $13 \mathrm{~Hz}, 35 \mathrm{~Hz}$ and $65 \mathrm{~Hz}$ without payload and $11 \mathrm{~Hz}, 33 \mathrm{~Hz}$ and $60 \mathrm{~Hz}$ with a $15 \mathrm{~g}$ payload. Previous experimental results have shown that the damping ratio of the flexible manipulator rangers from 0.024 to 0.1 [7]. The magnitudes and time locations of the impulses were obtained by solving equation (10) for the first three modes. 


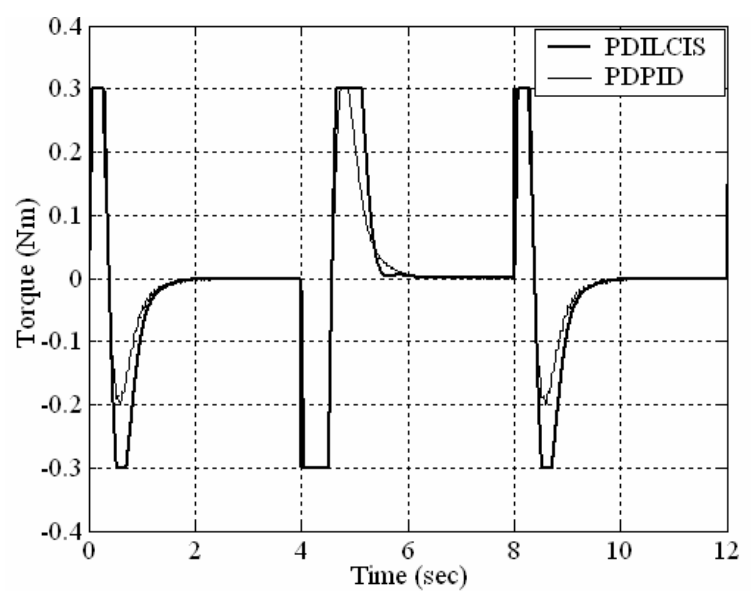

(a) Torque input (Time domain)

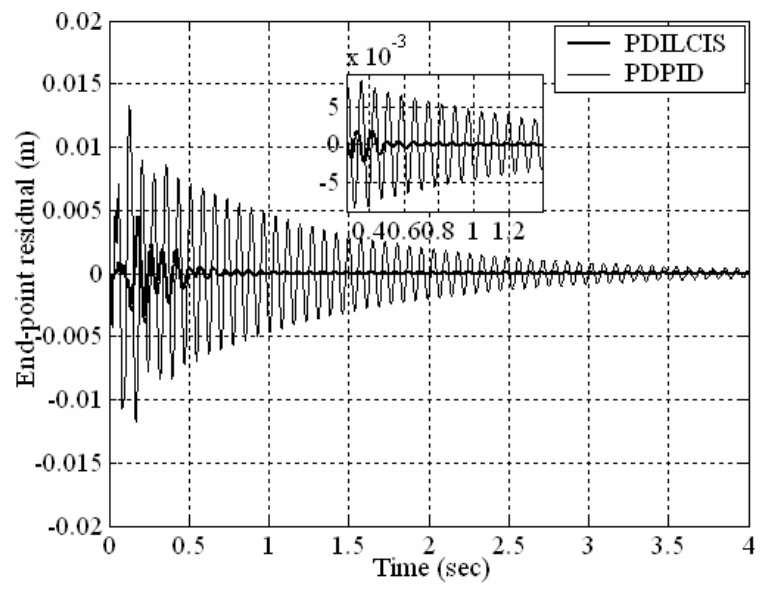

(c) End-point residual (Time domain)

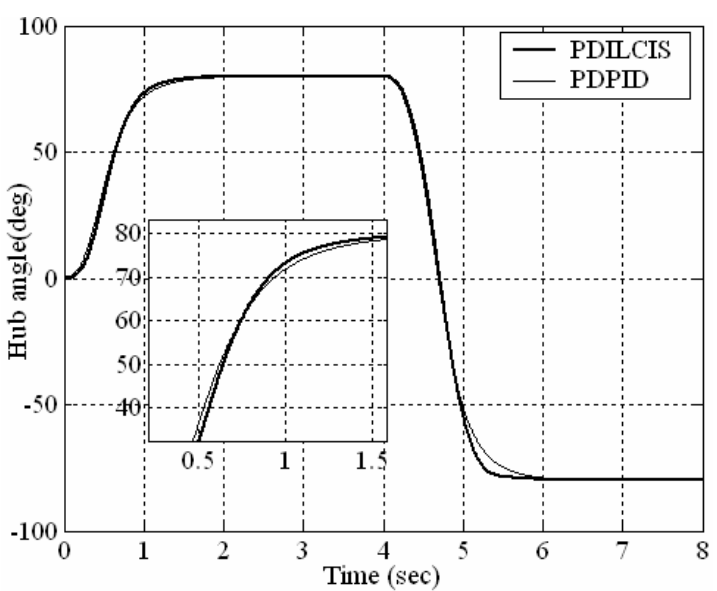

(b) Hub angle (Time domain)

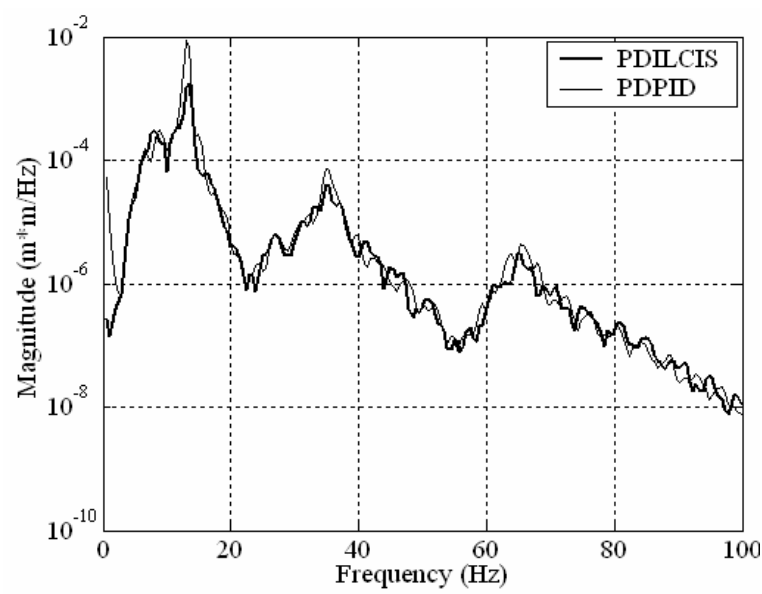

(d) Spectral density of end-point residual

Figure 17: Response of the manipulator with PD-ILC-IS and PD-PID control without payload

For digital implementation of the input shaper, locations of the impulses were selected at the nearest sampling time. In this case, the locations of the second impulse were obtained at $0.042 \mathrm{sec}, 0.014 \mathrm{sec}$ and $0.008 \mathrm{sec}$ for the three modes respectively. The developed input shaper was then used to pre-process the input reference shown in Figure 8. Figure 17 shows the resulting torque input driving the manipulator without payload with PD-PID and PD-ILC-IS control. It is noted that the proposed hybrid controllers are capable of significantly reducing the vibration of the manipulator. 


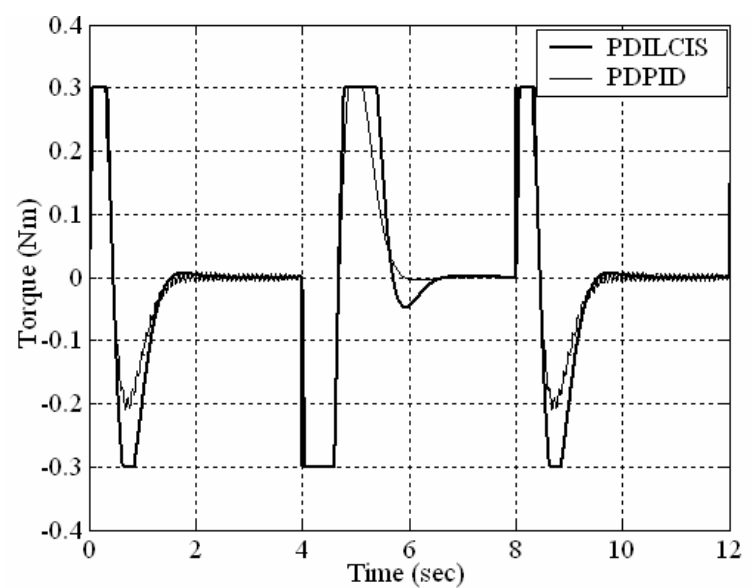

(a) Torque input (Time domain)

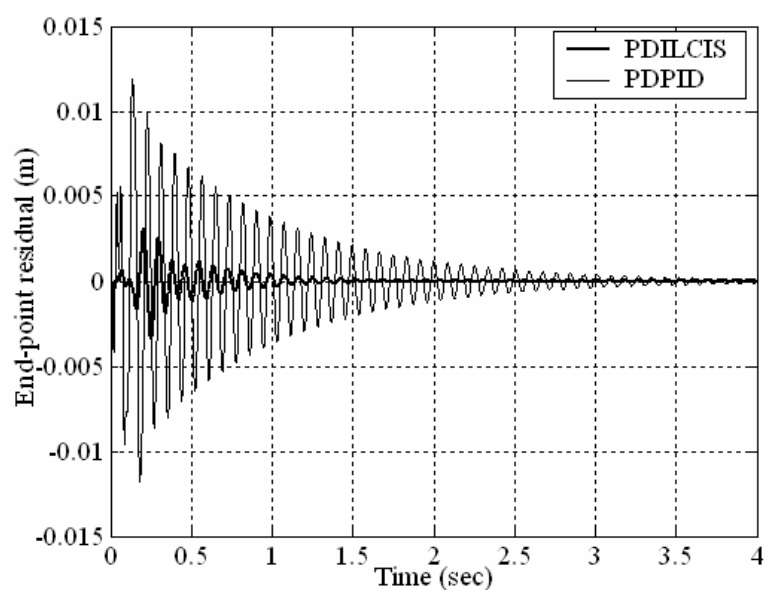

(c) End-point residual (Time domain)

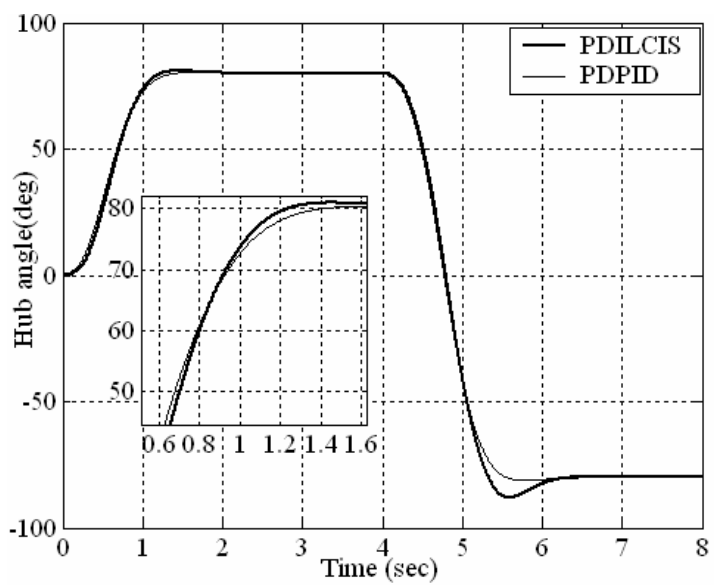

(b) Hub angle (Time domain)

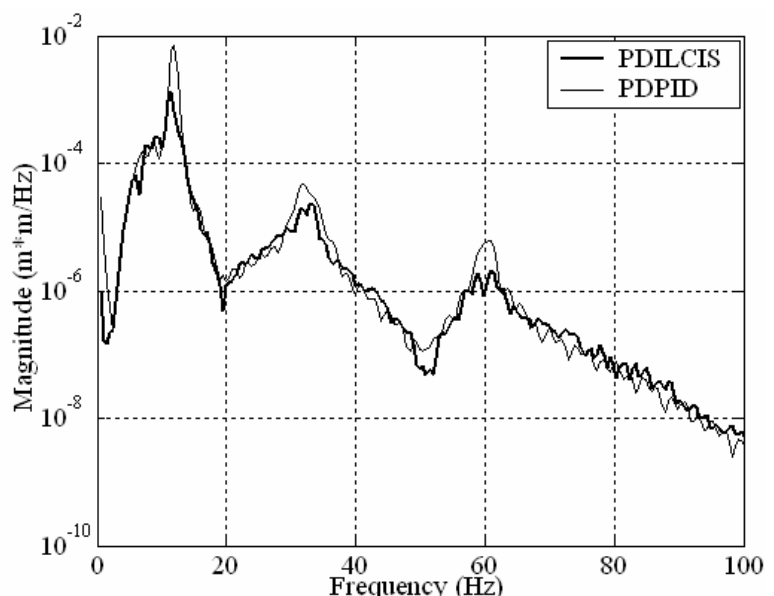

(d) Spectral density of end-point residual

Figure 18: Response of the manipulator with PD-ILC-IS and PD-PID control with $15 \mathrm{~g}$ payload

A significant amount of vibration reduction was achieved at the end-point of the manipulator with both control schemes. With PD-ILC-IS control, the maximum residual at the end-point was $\pm 0.015 \mathrm{~m}$. Moreover, the vibration of the system settles within $1.5 \mathrm{~s}$, which is twofold improvement as compared with PD-PID. This is also evidenced in the SD of the endpoint residual, which shows lower magnitudes at the resonance modes. For the manipulator with a $15 \mathrm{~g}$ payload, a similar trend of improvement is observed. The performance of the controller at input tracking control is maintained similar to PD-ILC control. Moreover, the controllers are found to be able to handle vibration of the manipulator with a payload, as significant reduction in 
system vibration was observed. Furthermore, the closed-loop systems required only $1.5 \mathrm{~s}$ to settle down. This is further evidenced in Figures 19 and 20, which show the level of vibration reduction with the end-point residual responses at the resonance modes of the closed-loop systems as compared to the open-loop system.

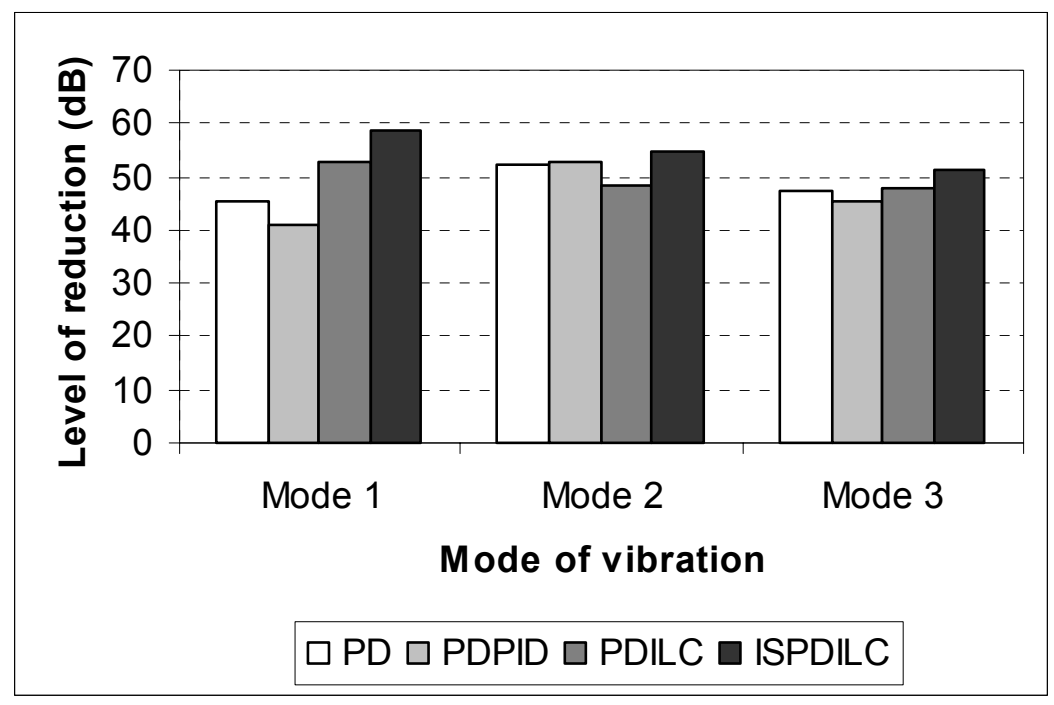

Figure 19: Level of vibration reduction with closed loop techniques as compared to open loop for the manipulator without payload.

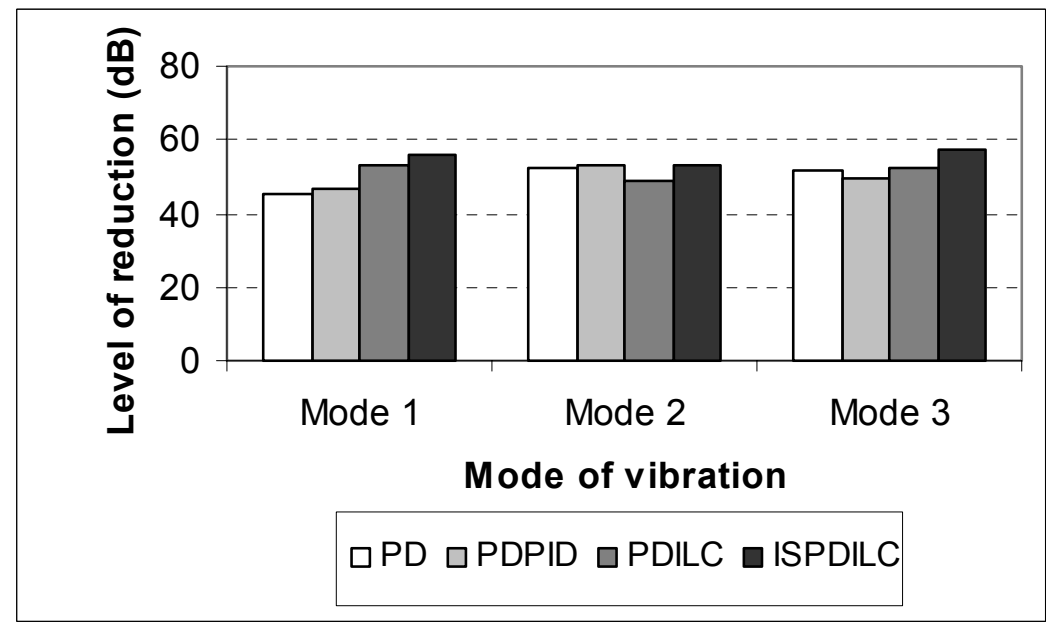

Figure 20: Level of vibration reduction with closed loop techniques as compared to open loop for the manipulator with a $15 \mathrm{~g}$ payload. 


\section{CONCLUSION}

The development of hybrid learning control schemes for input tracking and vibration suppression of a flexible manipulator has been presented. The control scheme has been developed on the basis of collocated PD with ILC based on GA optimization and input shaping. The control schemes have been implemented and tested within the simulation environment of a single-link flexible manipulator without and with a payload. The performances of the control schemes have been evaluated in terms of input tracking capability and vibration suppression at the resonance modes of the manipulator. Acceptable input tracking control and vibration suppression have been achieved with both control strategies. A comparative assessment of the control technique has shown that hybrid PD-ILC-IS scheme results in better performance than the PD-PID control in respect of hub-angle response and vibration suppression of the manipulator.

\section{REFERENCES}

[1] Book WJ, Majette M. Controller design for flexile distributed parameter mechanical arm via combined state-space and frequency domain techniques. Transactions of ASME: Journal of Dynamic Systems, Measurement and Control 1983; 105(4): 245-254.

[2] Piedboeuf JC, Farooq M, Bayoumi MM, Labinaz G, Argoun MB. Modelling and control of flexible manipulators - revisited. Proceedings of 36th Midwest Symposium on Circuits and Systems. (Detroit): 1983, pp.1480-1483.

[3] Yurkovich S. Flexibility effects on performance and control. Robot Control 1992; Part 8:321323.

[4] Tokhi M. O., Mohamed Z. and Shaheed M. H. Dynamic characterisation of a flexible manipulator system. Robotica, 2001, 19(5), 571-580.

[5] Mohamed Z. and Tokhi M. O. Vibration control of a single-link flexible manipulator using command shaping techniques. Proceedings of IMechE-I: Journal of Systems and Control Engineering, 2002, 216(2), 191-210.

[6] Gevarter, Basic relations for control of flexible vehicles, American Institute of Aeronaunting Astronaunting Journal, 1970, 8(4), 666-672. 
[7] Azad K. M. Analysis and Design of Control Mechanism for Flexible Manipulator Systems. $\mathrm{PhD}$ thesis, Department of Automatic Control and Systems Engineering, The University of Sheffield, 1994.

[8] Arimoto S., Kawamura S., Miyazaki F., "Applications of Learning Method For Dynamic Control of Robot Manipulators", Proceedings of $24^{\text {th }}$ Conference on Decision and Control, Ft. Lauderdale, (1985) pp. 1381-6.

[9] Arimoto S., Kawamura S., Miyazaki F., "Bettering Operation of Robots by Learning", Robotic Systems, pp. 123-140 (1984).

[10] Arimoto S., Kawamura S., Miyazaki F., "Convergence, Stability and Robustness of Learning Control Schemes for Robot Manipulators", Recent Trends in Robotics: Modelling, Control and Education, ed. By M. Jamshidi, L.Y.S. Luh, and M. Shahinpoor (1986) pp 307316.

[11] Moore K. L., Iterative Learning Control for Deterministic Systems, Advances in Industrial Control. Springer-Verlag, 1993.

[12] Mailah M., "Intelligent Active Force Control of A Rigid Robot Arm Using Neural Network and Iterative Learning Algorithm”, PhD Thesis, (University of Dundee, Dundee, 1998).

[13] Garg, D. and Kumar, M., “ Optimal Path Planning and Energy Minimization via Genetic Algorithm Applied to Cooperating Manipulators", Proceeding of ASME, Special Publication of DSCD/ASME, Paper No. IMECE2001/DSC-24509, 2001.

[14] Warwick K., Control systems: an introduction, (Prentice Hall, London, 1989).

[15] Singer N. C. and Seering W. P. Preshaping command inputs to reduce system vibration. Transactions of ASME: Journal of Dynamic Systems, Measurement and Control 1990; 112(1):76-82. 